\title{
Relaxation Times in Magnetic Nanoparticles System and Memory Effects
}

\author{
M. Osaci*, C. Abrudean and A. Berdie \\ Electric Engineering and Industrial IT Department, Faculty of Engineering \\ Hunedoara, "Politehnica Timisoara" University \\ Revolutiei no. 5, 331128 Hunedoara, Romania
}

(Received July 9, 2007; revised version October 18, 2007)

\begin{abstract}
Some memory effects in nanoparticle systems, similar to those seen in spin glass systems, may have important device applications, by tuning the interaction and the particle size. Recently, this subject provoked a special interest in nano-sciences. In this work we present a study, by simulation of the mode in which the behavior of a magnetic nanoparticle system is influenced by the superposition of the dimensions' distribution, the effective anisotropy constants and the disposal of nanoparticles in the sample, if we take into account the dipolar magnetic interaction.
\end{abstract}

PACS numbers: 72.15.Lh, 75.50.Tt, 78.20.Bh

\section{Introduction}

In the magnetization process, the stepped alignment of the magnetic moments in the field's direction can take place via two distinct mechanisms. In the first one, the magnetic moments remain fixed against the single-domain nanoparticle, and under the field's action, each nanoparticle rotates in such a way that the orientation of the magnetic moment gets closer to the field's direction. In the second one, the magnetic moments rotate against the nanoparticle, which remains fixed. The process by which the nanoparticle assembly gets back to the thermodynamic balance is called magnetic relaxation.

Due to these mechanisms, two relaxation processes appear [1]: the Brown relaxation, correlated with the nanoparticles rotation, and the Néel relaxation connected to the rotation of the magnetic moment inside the nanoparticle. In the case of a system with single-domain nanoparticles caught rigidly into a solid

*corresponding author; e-mail: osaci.mihaela@fih.upt.ro 
matrix, their rotation is blocked. In this situation, the field rotates only the orientation of the magnetic moment against the nanoparticle, thus the magnetic viscosity in such structures is due to the Néel relaxation processes $[2,3]$.

When the relaxation time $\tau$ is smaller than the measuring time, the nanoparticle system has a superparamagnetic behavior, otherwise the relaxation is blocked. Thus, due to the non-balance phenomena featured by irreversibility and hysteresis, in the nanoparticle systems appear magnetic memory effects, which are very important for applications in magnetic memory devices. In major speciality works [4-7], these effects are assigned to the appearance of the spine collective states featured by complex phenomena of spine-glass type. In this context the concept of blocking temperature $T_{\mathrm{B}}[4-7]$, as a temperature when the system is passing to the spine-glass state, was introduced. In this respect, when the system's temperature $T<T_{\mathrm{B}}$, the magnetic moments of the single-domain particles are frozen, e.g. they do not relax, and when $T>T_{\mathrm{B}}$, the magnetic moments are relaxing. There are also current works [8] which assign these behaviors to the distribution of the relaxation times as a result of the distribution of the nanoparticles' dimensions and the effects of dipolar magnetic interaction between them. Due to the distribution of the relaxation times, some are too small against the measuring time and some are too big, thus, at a given temperature, even from the interval $\left(T_{\mathrm{B}}, T_{\infty}\right)$ with $T_{\infty}$ high temperature, the magnetic moments of the small particles balance perfectly, whilst the magnetic moments of the big particles are blocked. In this context, the memorize effects could be influenced not only by the presence of dipolar magnetic interactions but also by the distribution of the sample's particle dimensions. This idea is verified experimentally on nikel ferrite samples [8], $\mathrm{NiFe}_{2} \mathrm{O}_{4}$, embedded into a $\mathrm{SiO}_{2}$ non-magnetic matrix in various concentrations, prepared by soil-gel technique. The Mössbauer measurements show that the dipolar magnetic interactions influence the magnetic ordering only locally and not in the entire sample's volume and, moreover, a slowing-down of the relaxation processes in the diluted sample was noticed, as in the case of the weak dipolar magnetic interaction between nanoparticles and even at the room's temperature.

In this paper we will study, by simulation, how the magnetic relaxation process in magnetic nanoparticles disperse systems is influenced by the superposition of three factors: dipolar magnetic interaction, dimensions' distribution, and the nanoparticles' effective anisotropy constants distribution.

\section{Model summary}

In order to study the dynamics of a magnetic moments system, we consider that at a given moment the action of the external magnetic field stops, and we monitor the behavior of the system's remanent magnetization in time.

We use the tridimensional simulation model presented in [9]. In this model, we assume that the spherical nanoparticles have an uniaxial anisotropy and are randomly distributed in the pre-set volume. The first stage of the method consists 
of the generation of the random values of the particle diameters and anisotropy constants. For this, we used the algorithm presented in [10-12]. The method of generating a lognormal distribution is based on the relations:

$$
d_{i}=\mathrm{e}^{\sigma_{\ln d} u_{i}+\ln d_{0}}
$$

for particle diameters, where $u_{i}$ are the values of a Gaussian distribution on $(0,1)$, with the average equal to $\operatorname{lnd}_{0}$ and the variance equal to $\sigma_{\ln d}^{2}$ and

$$
k_{i}=\mathrm{e}^{\sigma_{\ln k} u_{i}^{\prime}+\ln k_{0}}
$$

for particle anisotropy constants, where $u_{i}^{\prime}$ are the values of a Gaussian distribution on $(0,1)$, with the average equal to $\ln k_{0}$ and the variance equal to $\sigma_{\ln k}^{2}$.

The nanoparticles are randomly distributed in the considered volume. We assume that $\boldsymbol{r}_{i j}$ is the vector which joins the centers of the two particles $i$ and $j$. The $r_{i j}$ component is generated with the Box Mueller transform [9]:

$$
\left.r_{i j}=r_{\text {med }}+\sigma / \sqrt{(}-\ln (\operatorname{rand} 1)\right)[\cos (2 \pi \operatorname{rand} 2)+\sin (2 \pi \operatorname{rand} 2)],
$$

$\sigma$ is the variances of the $r_{i j}$ component.

The study of the particle system behavior begins in the moment when the external magnetic field action stops $(t=0)$. In these conditions, the overall energy of the system is the sum of the anisotropy energy and the magnetic dipolar interaction energy.

Let $\boldsymbol{M}_{i}$ be the magnetic moment of the particle $i$ and $\boldsymbol{M}_{j}$ the magnetic moment of the particle $j$. The magnetic dipolar interaction energy between two particles $i$ and $j$ is illustrated by the following relation [2,3]:

$$
\begin{gathered}
E_{i j d}=\varepsilon\left[\boldsymbol{m}_{i} \cdot \boldsymbol{m}_{j}-3\left(\boldsymbol{m}_{i} \cdot \boldsymbol{r}_{i j}\right)\left(\boldsymbol{m}_{j} \cdot \boldsymbol{r}_{i j}\right)\right] \\
\text { with } \varepsilon=\frac{\mu_{0}}{4 \pi} \frac{M_{i} M_{j}}{r_{i j}^{3}},
\end{gathered}
$$

$\boldsymbol{r}_{i j}$ is the unit vector of the direction which joins the particles $i$ and $j, \boldsymbol{m}_{i}, \boldsymbol{m}_{j}$, are the unit vectors of the magnetic moments of the particles $i$ and $j$, and $\mu_{0}$ is the vacuum magnetic permeability constant.

Considering the fact that the system particles have an uniaxial anisotropy, the overall energy of the $i$ particle formula is [2,3]:

$$
E_{i}=\sum_{j, j \neq i} E_{i j d}-K_{i} v_{i} \cos ^{2} \theta_{i}
$$

where $K_{i}$ is the $i$ particle uniaxial anisotropy constant and $\theta_{i}$ is angle between magnetic moment of the $i$ particle and anisotropy axis.

We consider the particular case when the magnetic anisotropy energy is much higher than the thermal energy $(\mathrm{KV} \gg \mathrm{kBT})$. In this approximation, these methods [9] use the Ising physical models (with two metastable states). Every particle $i$ belonging to the system has two possible stable states, separated by energy barriers $E_{\mathrm{bi}}$ used for calculation of the time unit probability to particle pass from a stable state into another stable state, $P\left(E_{\mathrm{bi}}\right)$. The relaxation time, 
for every particle of the system, is

$$
\tau_{i}=\frac{1}{P\left(E_{\mathrm{bi}}\right)} \text {. }
$$

The remanent magnetization, depending on the temperature, will be

$$
M_{\text {rem }}(t)=M(0) \exp \left(-\frac{t}{\tau}\right),
$$

where $\tau$ is a medium relaxation time and $M(0)$ is the magnetization in the moment when the outer magnetic field is released - the saturation magnetization.

\section{The relaxation times distribution considering the superposition of the nanoparticles' dimensions distribution and the dipolar magnetic interactions}

The simulations were performed on a system with 270 spherical fine particles of magnetite, with the saturation magnetization $M_{\mathrm{S}}=4.7 \times 10^{5} \mathrm{~A} / \mathrm{m}$. In the first phase we consider that the nanoparticles have the same anisotropy constant $K=19000 \mathrm{~J} / \mathrm{m}^{3}$ and that the particle dimensions have a lognor-
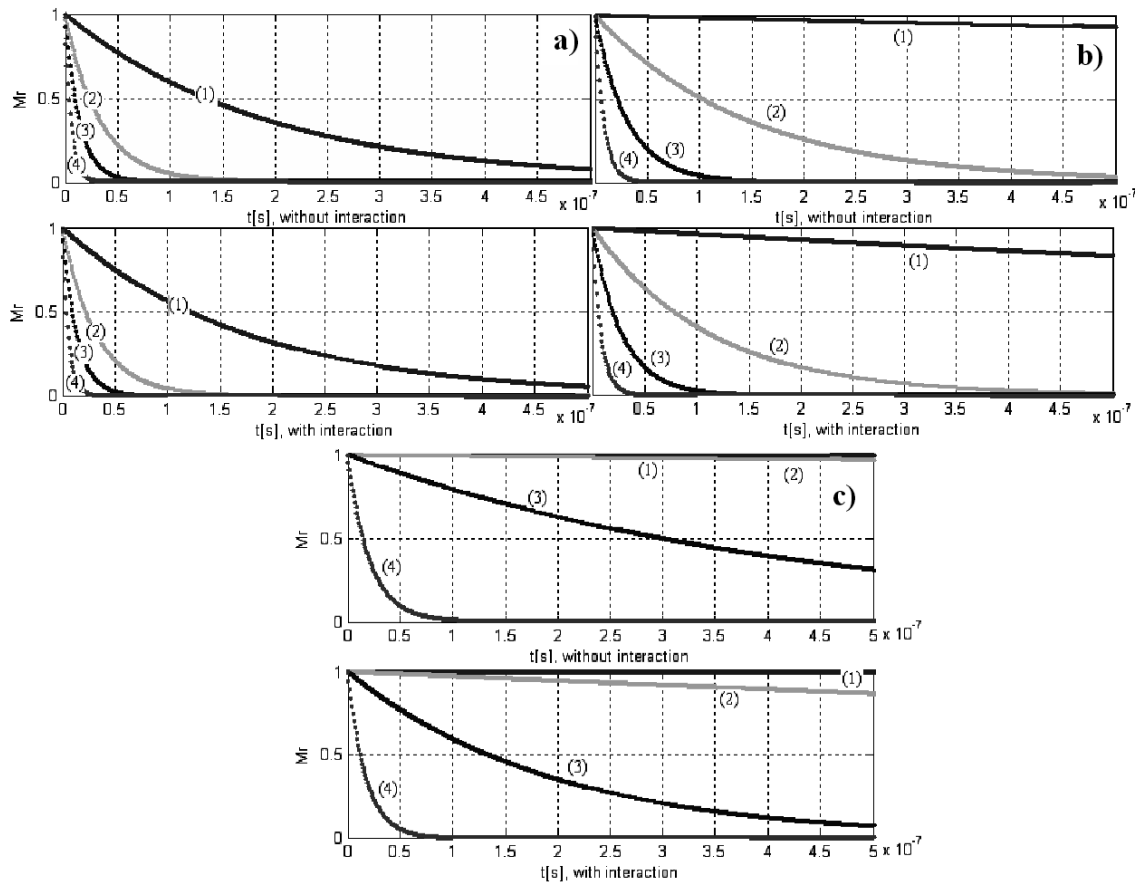

Fig. 1. The reduced remanent magnetization depending on time, for a system of magnetite fine particles (concentration $2 \times 10^{23}$ part $/ \mathrm{m}^{3}$ ), with $3 \mathrm{D}$ randomisation, into a pre-set volume without distribution of particle dimensions (a) and a random distribution of the diameters with $\sigma_{\ln d}=0.1(\mathrm{~b})$, and $\sigma_{\ln d}=0.15$ (c), at the following temperatures: (1) $100 \mathrm{~K},(2) 150 \mathrm{~K}$, (3) $200 \mathrm{~K}$, (4) $300 \mathrm{~K}$. 

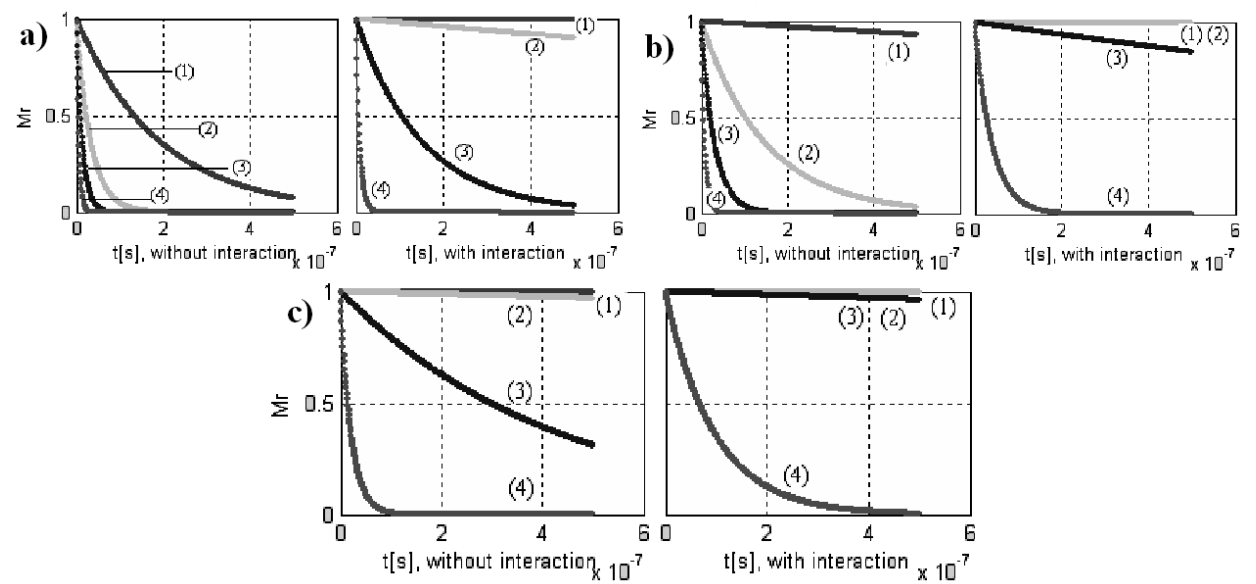

$\mathrm{t}[\mathrm{s}]$, with interaction $\times 10^{-7}$

Fig. 2. The reduced remanent magnetization depending on time for a system of magnetite fine particles (concentration $7.65 \times 10^{23} \mathrm{part} / \mathrm{m}^{3}$ ), with $3 \mathrm{D}$ randomization, into a pre-set volume without distribution of particle dimensions (a) and a random distribution of the diameters with $\sigma_{\ln d}=0.1$ (b), and $\sigma_{\ln d}=0.15$ (c), at the following temperatures: (1) $100 \mathrm{~K}$, (2) $150 \mathrm{~K},(3) 200 \mathrm{~K}$, (4) $300 \mathrm{~K}$.

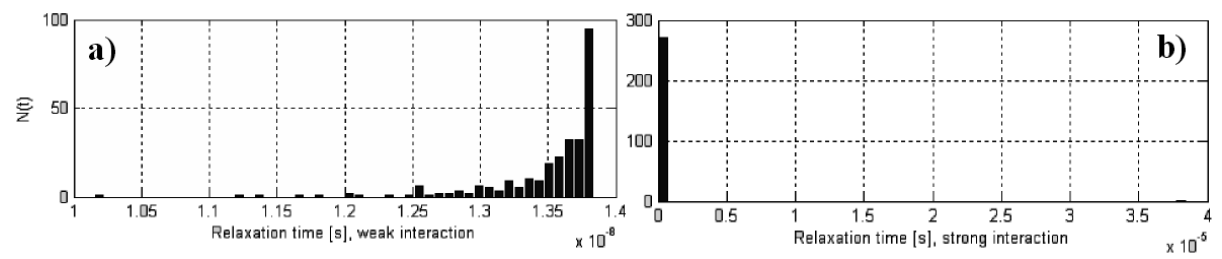

Fig. 3. Distribution of relaxation times due to the dipolar magnetic interactions between nanoparticles at a temperature of $200 \mathrm{~K}$ for a concentration of (a) $2 \times 10^{23} \mathrm{part} / \mathrm{m}^{3}$ (weak interaction) and (b) $7.65 \times 10^{23} \mathrm{part} / \mathrm{m}^{3}$ (strong interaction).

mal distribution. The most probable value of the particle diameter is $d_{\mathrm{mp}}=9 \mathrm{~nm}$ and we work for three values of the variance: $\sigma_{\ln d}^{2}=0.01$ and $0.0225\left(\sigma_{\ln d}=0.1\right.$ and 0.15$)$. We will study three cases: without interaction, weak interaction (a particle concentration of $2 \times 10^{23}$ part $/ \mathrm{m}^{3}$ ), strong interaction (a particle concentration of $7.65 \times$ $\left.10^{23} \mathrm{part} / \mathrm{m}^{3}\right)$.

The simulations are performed by using a distance distribution from the $i$ th particle having the variance $\sigma_{r j}=v_{r} r_{\text {med,j }}$ ( $j$ is the neighbors order) with $v_{r}=0.1$.

Figure 1 presents the evolution in time of the reduced remanent magnetization $M_{\mathrm{r}}(t)=M_{\mathrm{rem}}(t) / M_{0}$, with $M_{0}$ — the system's magnetization in the moment when the action of the external magnetic field stops, for four different temperatures of the system of $2 \times 10^{23}$ part $/ \mathrm{m}^{3}$ concentration. 

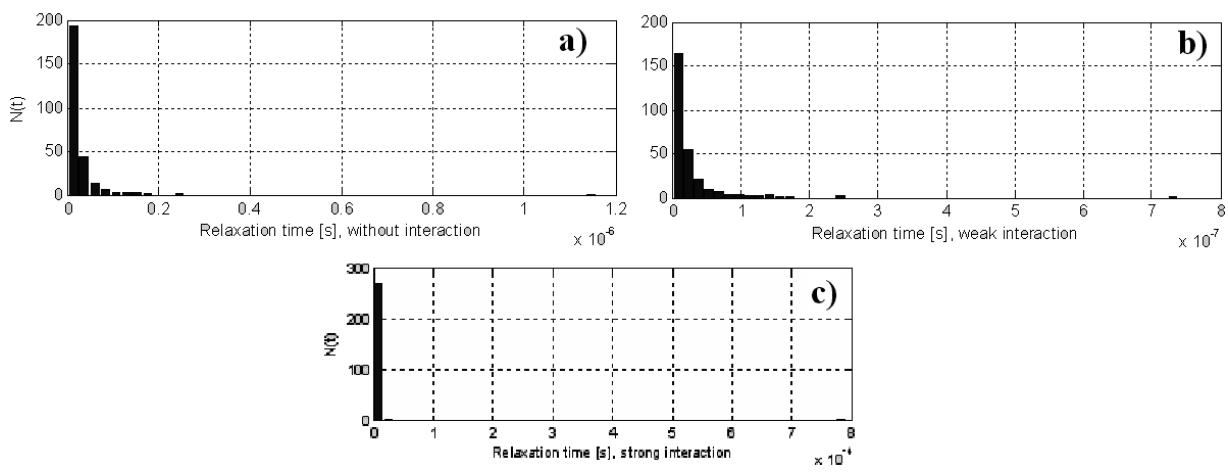

Fig. 4. Distribution of relaxation times due to the distribution of particles' dimensions $\left(\sigma_{\ln d}=0.1\right)$ dipolar magnetic interactions between nanoparticles at a temperature of $200 \mathrm{~K}$ without interaction (a), for a concentration of $2 \times 10^{23} \mathrm{part} / \mathrm{m}^{3}$ (b) and of $7.65 \times$ $10^{23} \mathrm{part} / \mathrm{m}^{3}$ (c).

One can observe that, in the case of low nanoparticles concentration $\left(2 \times 10^{23}\right.$ part $\left./ \mathrm{m}^{3}\right)$, e.g. at weak magnetic interactions between particles, the reduced remanent magnetization decreases significantly faster in time if we take into account the interactions, compared to the non-interactive case, but, in return, the dimensions' distribution strongly influences the variation in time of the reduced remanent magnetization, leading even to a blocking situation of magnetic moments at low temperatures (at $100 \mathrm{~K}$ and $\sigma_{\ln d}=0.15$ ) (Fig. 1c).

In the case of strong dipolar magnetic interaction between particles, e.g. high particle concentration $\left(7.65 \times 10^{23}\right.$ part $\left./ \mathrm{m}^{3}\right)$, the evolution in time of the reduced remanent magnetization is presented in Fig. 2. When the particles concentration in the sample is high, we can observe a more pronounced presence of the dipolar magnetic interaction. Thus, the reduced remanent magnetization decreases in time slower than for the non-interactive case, e.g. the average relaxation time increases by an increase in interaction's strength. If we also overlap the distribution of the particle dimensions over the effect of dipolar magnetic interactions, a much slower decrease in the reduced remanent magnetization in time is obtained, with blocking stages of the magnetic moments' orientation even at not very low temperatures (Fig. 2b and c).

We present in Fig. 3 the distribution of the nanoparticles' individual relaxation times due to dipolar magnetic interactions at a temperature of $200 \mathrm{~K}$, and in Fig. 4 the effects of the superposition of the dipolar magnetic interactions and distribution of nanoparticles' dimensions upon the relaxation times' distribution, at a $200 \mathrm{~K}$ temperature.

From the analysis of Figs. 3, 4 it results that the overlapping of the effects of the dipolar magnetic interactions and the distribution of the particles' dimensions leads to a displacement of the relaxation times' distribution towards higher values. It is interesting to see what is happening if upon these effects we also overlap the 
effect of the distribution of the particles' effective anisotropy constants (study from Sect. 4).

\section{The relaxation times distribution in the case of superposition of dimensions' distribution, of effective anisotropy constants, and of dipolar magnetic interactions}

In this section we also consider a lognormal distribution of the nanoparticles' effective anisotropy constants. This variation of the effective anisotropy constants is justified [13-16] by the appearance of a surface anisotropy of the nanoparticles, a structural anisotropy resulted from the discontinuity of the magnetic interactions between individual spines from the particle's surface.

In the magnetite nanoparticle system with a concentration of $2 \times$ $10^{23} \mathrm{part} / \mathrm{m}^{3}$ (weak dipolar magnetic interaction), we consider a lognormal distribution of the particles' diameter with the variance $\sigma_{\ln d}^{2}=0.0025\left(\sigma_{\ln d}=0.05\right)$, taking into account the dipolar magnetic interaction, and we consider a lognormal distribution of the effective anisotropy constants, with the most probable value of the effective anisotropy constant $K=19000 \mathrm{~J} / \mathrm{m}^{3}$ and the variance of the distribution which takes different values, i.e. $0.01,0.0225$, and $0.04\left(\sigma_{\ln K}=0.1,0.15\right.$, and 0.2$)$.

In the first stage, in order to have a comparison term for the introduction of the effective anisotropy constants' distribution, we consider the nanoparticles having the same effective anisotropy constant, but in return they show a distribution of the dimensions with the 0.0025 variance $\left(\sigma_{\ln d}=0.05\right)$. In Fig. 5 we present the relaxation times distributions of the considered nanoparticle system at a temperature of $200 \mathrm{~K}$.

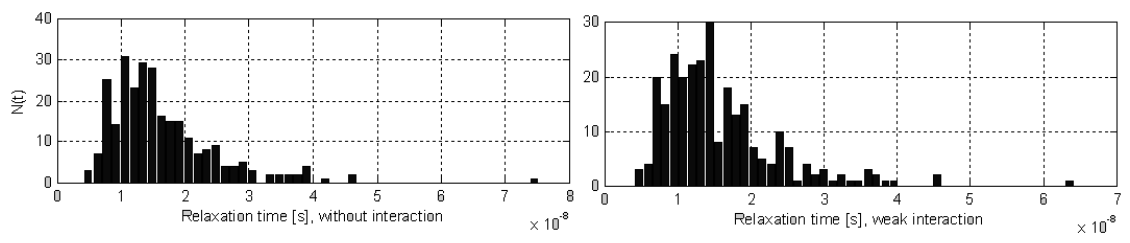

Fig. 5. Relaxation times distribution due to the particle dimensions distribution $\left(\sigma_{\ln d}=0.05\right.$ left $)$ and dipolar magnetic interactions between nanoparticles at a temperature of $200 \mathrm{~K}$ for a concentration of $2 \times 10^{23} \mathrm{part} / \mathrm{m}^{3}$ (right).

Figure 6 shows the histograms of the nanoparticles' relaxation times for the case when the variance of the dimensions distribution is kept, but the variance of the effective anisotropy constants' distribution increases.

From the figures obtained by simulation and presented above (Figs. 5, 6) we can see that the higher the variance of the effective anisotropy constants' distribution, the more the maximum of the relaxation times histogram moves towards higher time values, e.g the average relaxation time increases. 

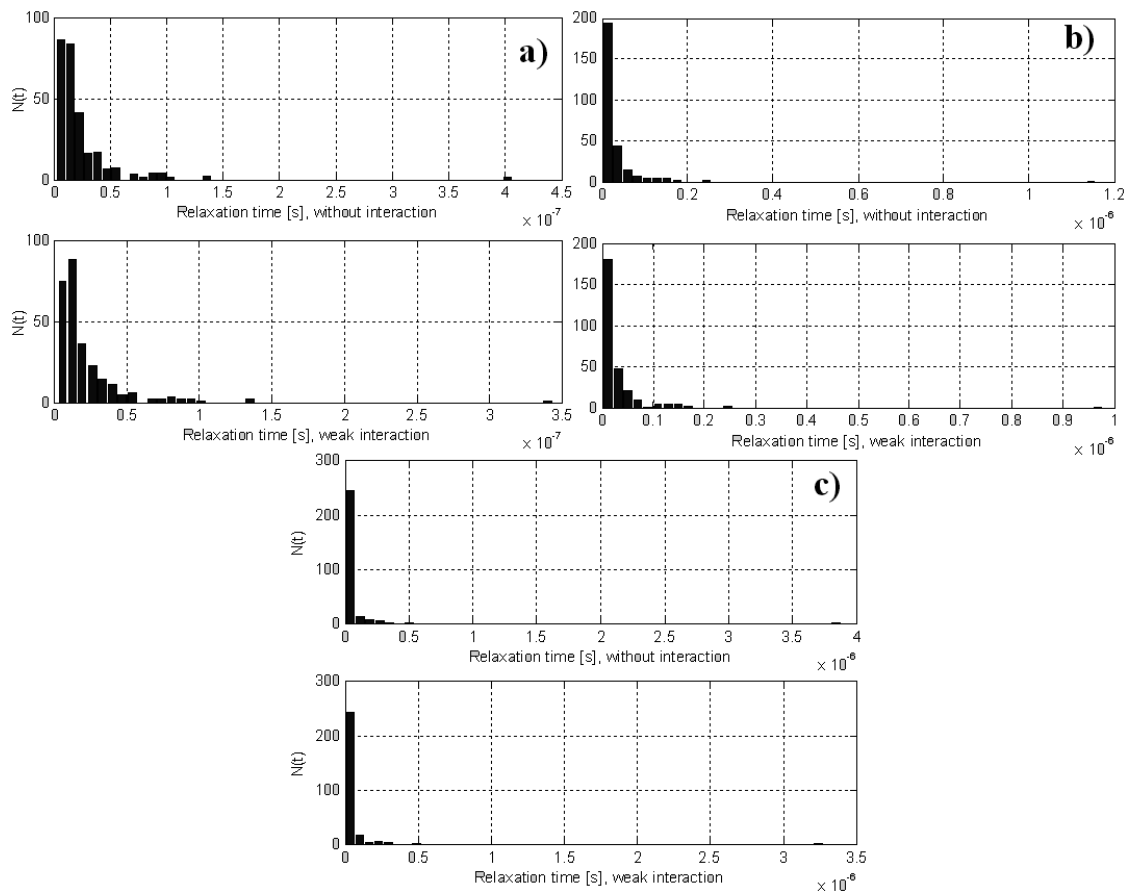

Fig. 6. Relaxation times distribution due to the particle dimensions distribution $\left(\sigma_{\ln d}=0.05\right)$, distribution of effective anisotropy constants ((a) $\left.\sigma_{\ln K}=0.1\right)$, ((b) $\left.\delta_{\ln K}=0.15\right),\left((\mathrm{c}) \sigma_{\ln K}=0.2\right)$, and dipolar magnetic interactions between nanoparticles at a temperature of $200 \mathrm{~K}$ for a concentration of $2 \times 10^{23}$ part $/ \mathrm{m}^{3}$ (low parts).

Figure 7 shows the dependence of the average relaxation time on the system's particle concentration at a $200 \mathrm{~K}$ temperature, for the considered magnetite nanoparticle system, when considering the superposition of the distributions of dimensions, effective anisotropy constants, and dipolar magnetic interactions. This dependence shows that for diluted systems the relaxation time decreases by increasing the concentration, reaches a minimum at a concentration of $5.7 \times 10^{23} \mathrm{part} / \mathrm{m}^{3}$, after which, for concentrated systems, starts to increase more by increasing the concentration.

Some experimental research studies of last period about the connection between the dipolar magnetic interaction strength and the relaxing time give us these results. Experimental measuring shows us, on the one side, that the relaxing time in such systems increases in the same time with the nanoparticle concentration decrease, which means it increases at the same time as the interaction strength's increases [12, 17-19], and on the other side, it shows a relaxing time decrease at the same time as the interaction strength increases [20].

Concerning the influence of the effective anisotropy constants' distribution of the nanoparticles, it can be noticed that as the distribution's variance increases, 


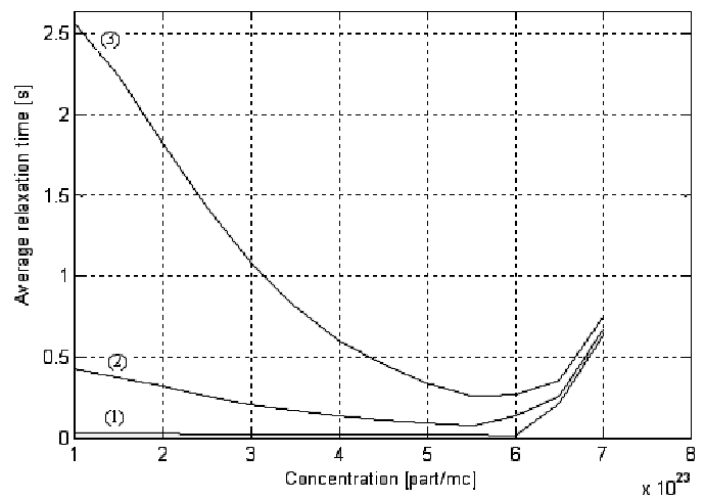

Fig. 7. Dependence of the average relaxation time of the particle concentration for a system of magnetite fine particles with a random distribution of the diameters $\left(\sigma_{\ln d}=\right.$ 0.1 ) at a $200 \mathrm{~K}$ temperature, (1) without effective anisotropy constant distribution; (2) with effective anisotropy constant distribution $\left(\sigma_{\ln K}=0.15\right)$; (3) with effective anisotropy constant distribution $\left(\sigma_{\ln K}=0.2\right)$.

the average relaxation time increases, the increase being more pronounced for the more diluted systems and less pronounced for the more concentrated systems.

\section{Conclusions}

In this work, a three-dimensional model was used for studying the superposition of the dimension distributions, the effective anisotropic constants, and the dipolar magnetic interactions for magnetic nanoparticle systems. In this model, the particles are arranged in a volume randomly, with a Gauss distribution generated with the Box-Mueller relation. This model is used to perform a simulation to determine the distributions for the relaxation times for weak and strong interactions at a $200 \mathrm{~K}$ temperature, the dependence of the medium relaxation time on the particles concentration at a $200 \mathrm{~K}$ temperature, and the dependence of the reduced remanent magnetization on time at different temperatures.

Considering the dipolar magnetic interaction between particles, one can observe that the average relaxation time is influenced by both the particle concentration and the particle volume. Generally, a decrease in the average relaxation time is obtained by increasing the particle concentration for diluted systems, and a pronounced increase in the average relaxation time is obtained by increasing the particle concentration for concentrated samples.

In addition, the distribution of the particle dimensions leads to a displacement of relaxation times distribution towards higher values, and by additionally considering a distribution of the nanoparticles' effective anisotropy constants, a significant increase in the relaxation times is obtained, especially for the more diluted systems. 
The results obtained in this work aim to complete the idea that these magnetic nanoparticle systems can be used for memory devices with special properties by the control of the dipolar magnetic interactions (modifying the distance between particles by modifying the particles concentration in the sample), by the control of the particle dimensions distribution and the effective anisotropy constants (through the technology of producing nanoparticles).

\section{References}

[1] R. Kotitz, W. Weitschies, L. Trahms, W. Semmler, J. Magn. Magn. Mater. 201, $102(1999)$.

[2] M.F. Hansen, S. Morup, J. Magn. Magn. Mater. 184, 262 (1998).

[3] J.L. Dormann, L. Bessais, D. Fiorani, J. Phys. C 21, 2015 (1988).

[4] R. Prozorov, Z. Zeshurun, T. Prozorov, Phys. Rev. B 59, 6956 (1999).

[5] P. Zhang, F. Zuo, F.K. Urban III, A. Khabari, P. Griffiths, A. Hosseini-Tehrani, J. Magn. Magn. Mater. 225, 337 (2001).

[6] D. Fiorani, J.L. Dormann, R. Cherkaoui, E. tronc, F. Lucari, F. D’Orazio, L. Spinu, M. Nogues, A. Garcia, A.M. Testa, J. Magn. Magn. Mater. 196-197, 143 (1999).

[7] R.W. Chantrell, N.S. Walmsley, J. Gore, M. Maylin, J. Magn. Magn. Mater. 196-197, 118 (1999).

[8] S. Chakraverty, M. Bandyopadhyay, S. Chatterjee, S. Dattagupta, A. Frzdman, S. Sengupta, P.A. Sreeram, Phys. Rev. B 71, 054401 (2005).

[9] M. Osaci, M. Panoiu, T. Heput, I. Muscalagiu, Appl. Math. Model. 30, 545 (2006).

[10] S.M. Ermakov, Monte Carlo Method and Related Problems, Technical Printing House, Bucharest 1976 (in Romanian), Nauka, Moscow 1971 (in Russian).

[11] I. Săcuiu, D. Zorilescu, Random Numbers, Applications in Economy, Industry and Study of Natural Phenomena, Academy Publ. House, Bucharest 1978.

[12] I. Mihoc, Probabilistic Calculation and Mathematical Statistics, Ed. UBB-Cluj-Napoca, 1994.

[13] F. Gazeau, J.C. Bacri, F. Gendron, R. Perzynski, Yu.L. Raikher, V.I. Stepanov, E. Dubois, J. Magn. Magn. Mater. 186, 175 (1998).

[14] O. Iglesias, A. Labarta, Phys. Rev. B 63, 184416-1 (2001).

[15] J.F. Hochepied, Online Nanotechnologies Journal 2, issue 1 (2001).

[16] M.V. Subbarao, S.M. Yusuf, R.G. Kulkarni, L.M. Rao, Pramana - J. Phys. 53, 341 (1999).

[17] P. Zhang, F. Zuo, F.K. Urban III, A. Khabari, P. Griffiths, A. Hosseini-Tehrani, J. Magn. Magn. Mater. 225, 337 (2001).

[18] J.L. Dormann, L. Spinu, E. Tronc, J.P. Jolivet, F. Lucari, F. D'Orazio, D. Fiorani, J. Magn. Magn. Mater. 183, L255 (1998).

[19] L.F. Barquin, R. Garcia Calderon, J. Phys. (Conf. Series) 17, 87 (2005), Fifth Intern. Conf. on Fine Particle Magnetism.

[20] S. Morup, E. Tronc, Phys. Rev. Lett. 72, 3278 (1994). 